\title{
Exploring the Sensed and Unexpected: Not Looking in Gaze Interaction
}

\author{
Argenis Ramirez Gomez \\ Lancaster University \\ United Kingdom \\ a.ramirezgomez@lancaster.ac.uk
}

\author{
Hans Gellersen \\ Lancaster University \\ United Kingdom \\ h.gellersen@lancaster.ac.uk
}

\begin{abstract}
Gaze interaction paradigms rely on the user needing to look at objects in the interface to select them or trigger actions. "Not looking" is an atypical and unexpected interaction to perform, but the eye-tracker can sense it. We illustrate the use of "not looking" as an interaction dynamic with examples of gaze-enabled games. We created a framework containing a spectrum of five discrete categories for this unexpected use of gaze sensing. For each category, we analyse games that use gaze interaction and make the user look away from the game action up to the extent they close their eyes. The framework is described based on whether specific game events mean the player might not; cannot; should not; must not; or does not look. Finally, we discuss the outcomes of using unexpected gaze interactions and the potential of the proposed framework as a new approach to guide the design of sensing-based interfaces.
\end{abstract}

\section{CCS CONCEPTS}

- Human-centered computing $\rightarrow$ Human computer interaction (HCI); Interaction design theory, concepts and paradigms;

\section{KEYWORDS}

Interaction Design; Gaze Interaction; Games; Design

ACM Reference Format:

Argenis Ramirez Gomez and Hans Gellersen. 2019. Exploring the Sensed and Unexpected: Not Looking in Gaze Interaction. In Proceedings of the Half way to the Future Symposium 2019 (HTTF 2019), November 19-20, 2019, Nottingham, United Kingdom. ACM, New York, NY, USA, 7 pages. https: //doi.org/10.1145/3363384.3363479

\section{INTRODUCTION}

The design of sensing-based interactions is often guided by the use of sensing technologies considering what is sensed and expected [2]. Benford et al. define what is sensed as what the computer system or sensor can measure. What is expected is referred to as the interactions the users might be expected to perform, and such interactions are natural to the interface. For example, the act of touching in a touchscreen or touchpad; speaking into a microphone; looking at a screen with eye-tracking sensing, etc. Unexpected interactions

Permission to make digital or hard copies of all or part of this work for personal or classroom use is granted without fee provided that copies are not made or distributed for profit or commercial advantage and that copies bear this notice and the full citation on the first page. Copyrights for components of this work owned by others than ACM must be honored. Abstracting with credit is permitted. To copy otherwise, or republish, to post on servers or to redistribute to lists, requires prior specific permission and/or a fee. Request permissions from permissions@acm.org.

HTTF 2019, November 19-20, 2019, Nottingham, United Kingdom

(C) 2019 Association for Computing Machinery.

ACM ISBN 978-1-4503-7203-9/19/11...\$15.00

https://doi.org/10.1145/3363384.3363479 are less usual and often performed when the interface is used in an atypical way or context.

Interfaces using the space of unexpected actions are uncommon and underexplored in Human-Computer Interaction (HCI) because they are ambiguous and create tension. One could think that not touching a touchscreen to select makes no sense. It might create an incompatible context that disrupts preconceptions of how the sensing technology is used [8], and yields unexpected interactions. On the other hand, Gaver et al. [8] see in this Ambiguity of Context an opportunity to enable designers to go beyond the limits of technology and to craft interactive designs that are engaging and thought-provoking.

Accordingly, we analyse gaze interaction with eye-tracking systems, exploring "not looking" as the unexpected interaction and incompatible context. We investigate the use of "not looking" mechanics in gaze-enabled game applications to define the space of unexpected gaze interactions. We reflect on the state of the art interaction dynamics to illustrate the "not looking" spectrum and provide a discussion of the lessons learned and the opportunities beyond play and gaze sensing.

Our work contributes a design framework developed on two levels. Firstly, it builds on the design of unexpected sensing-based interactions drawn from users' behaviour. These interactions are guided by the users deciding they might not; cannot; should not; must not; or do not interact as expected. Secondly, the framework defines the design space for "not looking" in gaze interaction.

\section{AMBIGUITY AND PLAY}

Applications using ambiguous sensing-based interactions are mostly represented in art-related, performative or playful contexts, to create a landscape of provocative and unconventional experiences inviting reflection [2]. In HCI, ambiguity is seen as a problem, but it is introduced as a resource for design [25]. The range of applications using the contextual ambiguity of the sensor is less crowded but present in examples like the POUTs [20]. Usually, pins are designed so the user can attach them to a pinboard, and it is unexpected for the pin to eject itself. POUTs are pins designed with this unexpected space of the interaction in mind: they pop out. This application created the opportunity to link physical and digital documents [21], which is useful, for instance, when removing a document in the digital space, the POUT will eject the documents from the physical pinboard.

Similarly, applications using gaze interaction are designed using eye-tracking technology information on where the user is looking. Therefore, what is unexpected is that the users stop looking. We take this opportunity to showcase how the use of ambiguity and 
exploration of the unexpected space of gaze sensing can create opportunities in the HCI design space.

Furthermore, ambiguity of context can be found in game applications and research. For instance, games using eye-tracking for interaction but penalise you for looking at the game objects [24, 34]; or a game that challenges the player to control two characters at the same time coordinating both halves of a single game controller[29]. Play offers ambiguity of context a safety net to explore the unexpected space of sensing-based interaction without disrupting the preconceived context by posing challenges. Given the unconventional use of gaze to "not look", we chose game applications that showcase this unexpected paradigm to analyse the space that it provides for interaction with eye-tracking systems.

Benford et al. [2] have already defined examples of unexpected interactions using the computer mouse, head-mounted displays or tangible interfaces, among others. They focused on different properties of movement or physical form of the interface, inviting designers to think of extremes, and bizarre scenarios. However, it is not obvious for the ubiquitous sensing of eye-trackers how those properties could be applied. To understand the space of unexpected gaze interactions, we created a framework illustrated by state of the art applications that make the user "not look" at the interface.

\section{DEFINING THE UNEXPECTED IN GAZE INTERACTION}

Gaze interaction is coupled with looking. Eye trackers provide information about where exactly the user is looking to enable interaction, for instance on a screen desktop, following the dynamic "What you look at is what you get" [12]. We look at the objects we want to interact with [38] because gaze signals interest [28]. Therefore, looking becomes the expected action in eye-tracking sensing technology.

Contrary to that, we can think about the other extreme of interaction and unexpected use of the sensor. An eye tracker can sense when and where we are looking, but also when we are not, and when the gaze signal is absent. However, not looking could take many forms, such as looking away with the eyes open; looking elsewhere; blinking, or closing our eyes.

In interaction, "not looking" is mostly associated with meditation apps in which users are asked to close their eyes, and they are guided through different exercises. In gaze interaction, not looking is an unexpected dynamic, only used in accessibility contexts with eye blinks to indicate a gaze selection [13,37]; or to "look away" coupled with behaviour metaphors in games to avoid eye contact with avatars [5].

Nevertheless, in gaze-enabled games, not looking is used to provide novel playful experiences. In the following sections, we analyse a set of games with gaze interaction dynamics. We propose five discrete categories in a framework based on the spectrum between directly looking to interact and the opposite extreme.

\section{WHAT YOU LOOK AT IS WHAT YOU GET}

Eye gaze interaction leverages the use of gaze as a natural pointer to trigger events by aligning where our eyes focus on the screen with objects of interest. In games, gaze interaction has been used to replace or complement controllers $[1,11]$ explicitly, or implicitly control the movement and effects of the game camera view $[9,18]$.

Just by looking, players can tag enemies [31]; aim weapons [7, 27]; fire [17]; signal which objects in the scene they want to select [4, 37], and make their character move to where they are looking [26, 27]. Gaze pointing is enough to trigger those explicit outcomes, but it could also be used to indicate the object of interest and confirm the selection with hand gestures [3]; voice [22, 32]; keyboard; mouse, or touch [16, 23].

When looking directly, gaze interaction becomes explicit to the user, and the effects that are triggered are expected and desired outputs of the interaction. For example, when players look at an enemy to attack, they are aware of what is going to happen. On the other hand, the effects of implicit gaze are not noticeable, because the change has no consequences on the gameplay. E.g., blurring the scene where the player does not look for realistic graphics rendering fostering immersion [9].

\section{NOT LOOKING: FIVE CATEGORIES}

The looking to not looking spectrum could be considered binary, but it can also be explained in different categories. "Not looking" as a concept can mean different things: not being able to look because it is physically impossible; looking away or elsewhere; blinking, or keeping your eyes shut. We illustrate in the following framework five discrete categories based on interactive gaze-enabled game designs. They demonstrate a range of potential interaction dynamics that play with the spectrum defined from looking to its unexpected use of the eye-tracking sensor (see Figure 1). In the framework, we describe examples of games and reflect on their position in the "not looking" space from directly looking for interaction to closing your eyes. In line with the definition of unexpected interactions, we define each category based on the users' decision to look away and their behaviour.

\subsection{First: Gaze Effects (Might not look)}

The Royal Corgi [36]. In the game, the player needs to network and talk to the king's counsellors to win their favour and become the royal corgi instructor. The game is presented in a first-person view and uses the keyboard to navigate through the different dialogue options, but where the player looks influences the character's reactions. Therefore, the player needs to be careful and pay attention to where they look not to upset the character with whom they interact. E.g., when talking to the Military Advisor, players need to show respect by maintaining eye contact. Moreover, when talking to the Budget Advisor, they need to be careful and not glance at his wife, or he will get offended.

Dying Light [30]. This example is a survival horror and adventure game set in a dystopian post-apocalyptic world. The player needs to infiltrate a quarantine zone while battling human enemies but also zombies. In the gaze-enabled version of the game, the player can aim firearms at gaze; automatically climb where they look and control a flashlight with gaze; all while moving around zombies. However, zombies will notice your presence when they are looked at, making them responsive to gaze interaction.

These two examples demonstrate that looking can create unintended outcomes. We look to explore the scene, and we also use 
Exploring the Sensed and Unexpected:

Not Looking in Gaze Interaction

HTTF 2019, November 19-20, 2019, Nottingham, United Kingdom Expected use
of the sensor
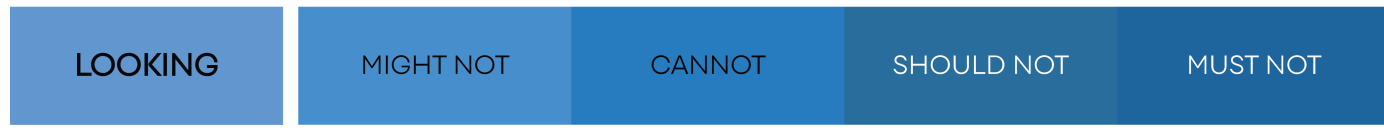
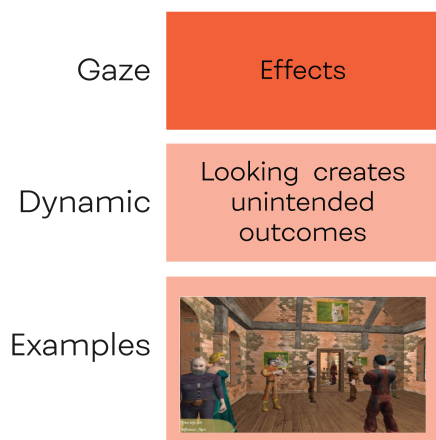

The Royal Corgi Vidal et al., 2015

Dynamic

Examples

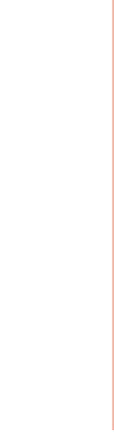

Result

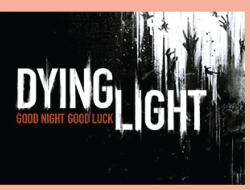

Dying Light Techland, 2015

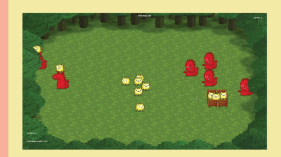

Shynosaurs Vidal. 2014

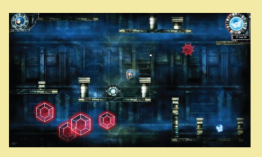

Limus and the eye of the Beholders Lankes et al., 2014

Attention Dilemmas
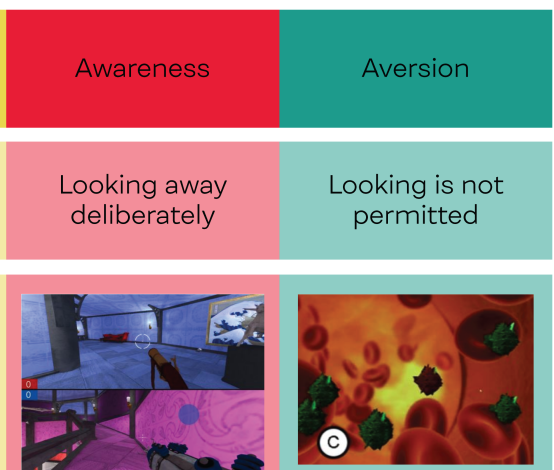

Virus Hunt

Screencheat Lankes et al., 2016

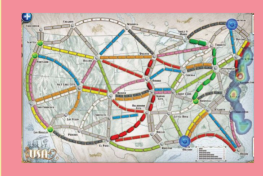

Ticket to Ride Newn et al., 2018

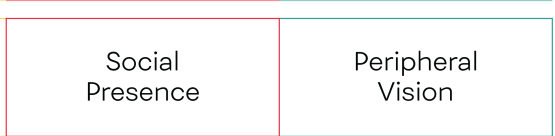

Unexpected use of the sensor

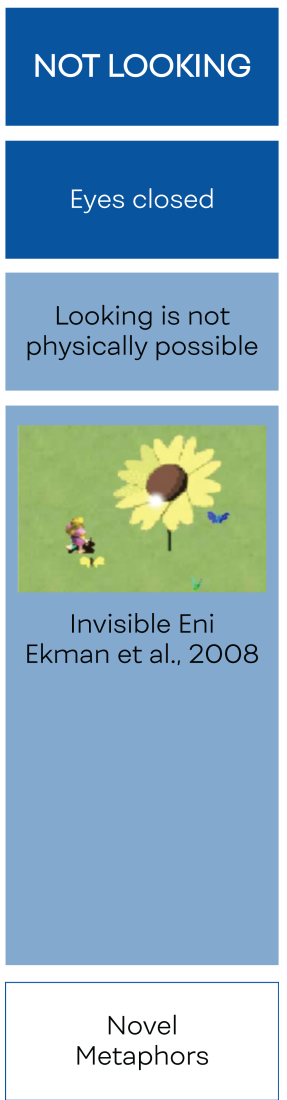

Figure 1: Unexpected Gaze Interaction Framework defining in 5 categories the spectrum from looking to "not looking". Each iteration is illustrated by examples that showcase the use of gaze, their looking dynamic, and the outcome of the paradigm.

looking for interaction (Dying Light). However, characters react to our attention and looking behaviour triggering what could be unwanted and unintended consequences. Accordingly, we might decide to stop scanning the scene and stop looking around not to promote these undesired gaze effects. In The Royal Corgi we might decide to only look at the character we are talking to when we notice they get upset when we look elsewhere, hence not looking at the scene. Similarly, in Dying Light, we might choose not to glance around in a space full of zombies, and keep our gaze low, to not draw the dying's attention to you.

\subsection{Second: Gaze Attention (Cannot look)}

Shynosaurs [35]. In Shynosaurs, the player needs to save the cuties by dragging and dropping them into safety with the mouse. During the task, the shynosaurs (dinosaur-like enemies) come from the woods to take the cuties away. However, if the player glances at the shynosaurs, they will feel intimidated and act like a shy naughty kid. They will stop and pretend they are not doing anything wrong.
The longer the player looks at them, the shyer they become, until they cannot stand being looked at and run away crying.

Limus and the eyes of the Beholders [15]. The hero, Limus, needs to escape a dark cellar by using a magic portal. The challenge is that the enemies called Spikees will try to catch Limus when they see him, going towards his position. However, if Limus looks at the Spikees, they will freeze and stop moving. In the game, the player controls Limus with a gamepad, whereas gaze interaction is used for the player to look at the Spikees. To make this chasing enemies stop, both the avatar (Limus) and the player need to look at them, disrupting the task of getting to the end of the level.

These two examples illustrate how looking can be challenging because we cannot look at two things simultaneously, introducing playing with "attention dilemmas". Both in Shynosaurs and Limus' games, the player faces a challenge in gaze attention and a constant dilemma. They need to either solve the main task: saving cuties or reaching the end of the level; or looking at the enemies to freeze them. This "not looking" dynamic makes the users stop looking at the game scene and focus their attention on the enemies. Although 
this dynamic might be difficult to extrapolate to other less playful domains, it offers an insight into how playing with this level of not being able to look can create greater awareness of the user's gaze attention and how to balance it.

\subsection{Third: Gaze Awareness (Should not look)}

Screencheat [14]. In this competitive split-screen First-Person Shooter multiplayer game, players are invisible and need to look at each other's screens to win. Each player needs to find the other and kill them first. To do this, they are encouraged to look at the half of the screen where the other player is playing. In the scene, they can see where the player is but also where they are looking.

Ticket to Ride [19]. Ticket to Ride is a board game in which players need to build train routes between cities across North America to gain points. Target routes are predefined by goal connection cards that are only visible for the corresponding player, and if incomplete will make the players lose points. The challenge becomes planning train networks carefully to minimise the risk that the opponent will block the path and take over the route. In the gaze-enabled digital version of the game, the gaze point is visualised to the opponent, spoiling the player's strategy.

In Screencheat the player is forced to look somewhere else to figure out where the other player is and win. However, the opponent player knows where you look in the game and might react accordingly. Similarly, in Ticket to Ride, the player is aware that the other player can see where she is looking at, and might decide to fool and deceive the opponent by looking somewhere else. Both examples show how the player knows that the opponents are aware of where they are looking. As players, we might decide that we should not look at the scene, not to spoil our strategy. Therefore, we might choose to look away deliberately to trick the other player.

\subsection{Fourth: Gaze Aversion (Must not look)}

Virus Hunt [34]. In this arcade game, players win points by removing viruses by touching them on the screen. However, if the player looks at the virus, it duplicates, spreading the infection.

SuperVision [24]. This game is a collection of three mini-games exploring the use of gaze aversion and peripheral vision. In the games, players need to overcome mouse manipulation tasks and challenges in perception in peripheral vision of objects with different size; colour; and form. When players look at the game objects, they are penalised, and they are forced to look away and work only with what they can see in their peripheral vision.

These two game examples are designed to explore two main concepts: touch or mouse manipulation, and the use of peripheral vision with gaze interaction. Further, SuperVision expands on just playing with what can be seen in the periphery, and the three mini-games leverage perception capabilities by proposing visual challenges in peripheral vision. Both SuperVision and Virus Hunt establish rules that make the players stop looking or look away. The player is penalised when looking at the objects in the scene that need to be sorted by using gaze aversion dynamics. In other words, looking is not permitted, but it is still possible (it is not required to close the eyes). Through the games, players are unsuccessful at overcoming the challenges, because the eyes are attracted to objects that pop up. They might look and fail, making them understand that to succeed, they must not look but need to figure out how to do things by looking elsewhere.

\subsection{Fifth: Gaze Absence (Eyes Closed)}

Invisible Eni [6]. This game uses gaze interaction; pupil dilation (size); and blinking, to affect different game states. The goal of the game is to move Eni close to butterflies so she can guide them to flowers and feed them with nectar to be free. Eni must complete this task avoiding the nightmare monsters that chase her. However, when the players close their eyes, Eni does too, and disappears into a puff of smoke, evading the monsters and protecting herself.

This last example illustrates the extreme of not looking that happens when the user shuts their eyes, and the eye-tracker loses the gaze information. Overall, looking is not physically possible, because we cannot see with our eyes closed. Because of how unexpected closing one's eyes is in gaze interaction, there are not many examples that showcase this category of "not looking".

\section{BEYOND PLAY: LESSONS LEARNED}

Our exploration of the unexpected space of gaze interaction through new playful game dynamics helped to shape opportunities that could be extrapolated in other genres and applications within the HCI field. Figure 1 extends on the defined framework towards the design of unexpected gaze interactions. It shows each category in the "not looking" spectrum; how they relate to the type of gaze concept; the game dynamic illustrated in the examples; and the resulting generalised themes.

\subsection{Social Gaze and Awareness}

The games using gaze effects, introduce to some extent the Midas Touch [33] during gaze pointing. This is related to the trigger or selection of objects when we look at them without the intention to interact with them. In HCI, the Midas Touch is a problem and it is often avoided. For example, in desktop applications in which to select with gaze, we would use the eyes to indicate the object of interest and another input, such as the keyboard, to confirm the selection. In games, it can be leveraged, not only to introduce dynamics for not looking but also to introduce the use of Social Gaze. The Royal Corgi [36] uses social gaze behaviours in a virtual world that led to more practical applications of gaze and greater immersion in a playful experience. However, Social Gaze is not unique to the gaming context. For instance, in 3D virtual world online communities, or meeting spaces of the future, gaze behaviour could be useful to recreate real-life situations. E.g. users could know if they are looked at; where another user is looking at; or avoiding to make eye contact to not engage in starting a conversation accordingly.

On the other hand, in both Screencheat [14] and Ticket to Ride [19], gaze visualisation is presented as a way to share the opponent's experience, and it has been demonstrated to have a positive effect in social presence [14]. Moreover, although the examples are set in a competitive context, this visualisation could also be used to add a new layer of non-verbal communication between two parties. This showcases that gaze could be used in the digital space, e.g. in remote collaborative work-spaces. Further, this category in the "not looking" framework allowed players to predict and try to understand their opponent's strategy and behaviour. Gaze patterns are a 
Exploring the Sensed and Unexpected:

Not Looking in Gaze Interaction

good asset for systems to predict intentions [10]. However, systems need to be aware that such intentions might not always be real, and the user could also try to "fool" when aware that she is being observed. This presents opportunities, not only to explore deciding not to look in the play space but also to consider this possibility to train the recognition systems of the future.

\subsection{Peripheral Vision and Metaphors}

The use of gaze aversion allowed the exploration of peripheral vision perception in games, leading to potential applications to train peripheral awareness [24]. Greater peripheral awareness can be useful, for example, to pick up notifications without looking at them. In a way, this paradigm could also solve the "Attention Dilemma" posed in Shynosaurs [35], as the player might be able to focus their attention on the enemies while performing the task in peripheral vision. Moreover, using gaze aversion as the "not looking" gaze interaction dynamic could train users to resist the impulse of looking at things that pop up. Playing with "not looking" could train inhibition control [24]. In turn, this could guide the design of the interfaces of the future that do not disturb the user from the main task. For example, GPS and navigation systems that do not need to be looked at, creating safer driving environments.

On the other hand, "not looking" enhanced the use of metaphors to guide the gaze interaction through the narrative of the game. In Invisible Eni [6], when the tracker loses the eyes once they are closed, the player character disappears. This example uses blinks as a means or metaphor to escape dangerous situations or to close the eyes to protect ourselves from intimidating events. In other words, not looking by closing the eyes creates new design opportunities to explore gaze metaphors. For instance, closing one's eyes to avoid danger as we would do in real life; or a "blind faith" that with our eyes shut, nothing wrong can happen, and we need to trust the system. Other examples could use closing the eyes as a sign for concentration; resting, or to disappear from the scene for a moment. New metaphors could guide the design of future applications, for instance, for meditation. In meditation apps, the user is guided to close their eyes, but detecting the action could signal that the user is ready to trust the system to guide them, and wait for further instructions. On the other hand, opening one's eyes could signal the system that we need to do something else and have a break. Overall, novel metaphors considering the "not looking" space can inform future systems to customise the experience based on users' behaviour.

Moreover, metaphors are not exclusive of the fifth category of the framework but present throughout the different examples of the spectrum. "Not looking" created the space for new gaze interactions guided by a narrative, such as looks that challenge or defy or looks with social meaning [36]; looks that intimidate [35]; looks that freeze [15]; and looks that could petrify, kill or charm [24]. This showcases the potential of exploring the spectrum of the proposed framework for interaction design.

Overall, exploring the unexpected space of gaze interaction in playful scenarios has led to solutions that could influence the future design of other application genres. However, it is not clear how to generalise the different categories of the spectrum defined and how they could be applied to other sensing-based interactive systems.
HTTF 2019, November 19-20, 2019, Nottingham, United Kingdom

\section{DISCUSSION}

\subsection{Understanding Looking Away}

Based on the defined categories, we could understand the unexpected use of gaze interaction as a framework based on the users' decision not to look and modulation of their gaze behaviour. Each category of the spectrum must not be treated in chronological order but as a set of dynamics that move towards the complete absence of sensed gaze input. Nevertheless, between them, we can identify a consistent pattern: the user is in control of looking away, while the game engine is in control of the challenge and influences the user's decision to look elsewhere. We can define two sets of behaviours. First, when the users might realise through reflection on the game outcomes that they might not, cannot and should not look at the game scene (A). Second, the scenarios in which the game system pushes the users to look away, either with rules that they must obey or mechanics that make them stop looking (B).

One take of the first group (A) is to consider them closer to the space of the expected looking interaction. It is the game engine that introduces an effect or a challenge to gaze interaction. On the other hand, the user decides that looking is no longer suitable for the game context, and therefore falls on the "not looking" paradigm. Although in the second category (cannot look), the system is designed so the users cannot attend to simultaneous tasks, it is still the user who decides to look away and balance the attention to the different challenges of the game.

On the other hand, the second behaviour (B) introduces less flexible rules, posing challenging gaze interactions that present looking away as a "must" to be successful in the experience. Moreover, the users are also introduced to metaphors that require them to stop looking and to close their eyes. Both actions are the users' will, but the game engine challenge can influence them. They no longer offer an open space for reflection and decision but set the behaviour as a rule.

In contrast to the definition of what is sensed and not expected, current applications using gaze interactions and the ambiguity of looking away are not defined by the atypical use of the interface. This could be sometimes encouraged by the system itself. "Not looking" could either be a decision of the player created by the interface outcomes to the users' gaze, or a direct consequence of the application's rules. Therefore, "not looking" as an unexpected interaction could be originated in design; and designers could follow the proposed framework to trigger this not so "unexpected" users' behaviours.

\subsection{Designing Unexpected Interactions}

Although the different categories of the presented framework are centred around applications of gaze interaction, it is not clear how to generalise them to define the unexpected interactions space for other HCI genres. Moreover, whether the proposed categories are suitable to be used to design applications and interfaces with sensors beyond eye-tracking could shape the research agenda for this new design approach. We can only hypothesise how the framework can illustrate new uses of sensors for interaction.

Firstly, we could think of unexpected interactions with sensors. For instance, not moving or shaking a device with an accelerometer; not pressing a button; not speaking into a microphone; not touching 
a touchscreen with your fingers or a pen. Secondly, each stage of the spectrum could be different to each sensor, and it is up to the designer to introduce the effects to trigger the unexpected behaviour in each category.

We can take as an example pressing a button. Not pressing it might be applied in an interface that requires pushing it consistently and then a release for interaction (e.g. Dead man's switch). This system could also be considered a somehow switched input binary state, where pressing is the null signal. The button could also be inside a box, making it physically impossible to be pressed. Further, a red button with an alarm sign might suggest to the user that they must not press it. On the other hand, another one with a "please do not press" sign could be an instance of a button that should not be pressed. Moreover, the designer can think of situations in which the user might not press the button (when it is required). Maybe it is because it makes an uncomfortable noise, or because it gives you a low and not dangerous static shock. Another example could tackle a button that cannot be pressed. It might be a broken button; one that moves away; or one that pushes itself when the hand approaches.

Similarly, the lack of pen input in a tablet could lead to the design of mid-air gestures that might indicate the users' intention when they might not touch the surface, such as in a sorting task or an option selection. Moreover, we could also think of voice sensing systems that you need to whisper or even be silent to activate them. More pragmatically, silence could be the indicator of written spaces in speech-to-text systems. These examples could illustrate, for instance, learning opportunities for interfaces using buttons that fail; or novel pen and touch gestures on touchscreens. However, the framework and defined spectrum might also need to be fine-tuned for each technology.

Whereas this approach is useful when designing gaze-enabled interfaces, further research is needed to validate and generalise the model for other sensing technologies. Overall, the presented framework encourages designers to think with a focus on the users' behaviour in the unexpected interaction space.

\section{CONCLUSION}

We explored the concept of "not looking" as an ambiguous and atypical way of using eye-tracking technologies for gaze interaction. We defined a framework to design unexpected gaze interaction that could potentially be generalised to other sensing technologies. This offers a new design approach based on system rules and users' behaviour; and a potential tool for designers to explore the limitations of the technology and their implications.

\section{REFERENCES}

[1] Richard Bates, Stephen Vickers, and Howell O Istance. 2010. Gaze interaction with virtual on-line communities: levelling the playing field for disabled users Universal Access in the Information Society 9, 3 (2010), 261-272.

[2] Steve Benford, Holger Schnädelbach, Boriana Koleva, Rob Anastasi, Chris Greenhalgh, Tom Rodden, Tom Rodden, Jonathan Green, Ahmed Ghali, Tony Pridmore, et al. 2005. Expected, sensed, and desired: A framework for designing sensingbased interaction. ACM Transactions on Computer-Human Interaction (TOCHI) 12,1 (2005), 3-30

[3] Marcus Carter, Joshua Newn, Eduardo Velloso, and Frank Vetere. 2015. Remote gaze and gesture tracking on the microsoft kinect: Investigating the role of feedback. In Proceedings of the Annual Meeting of the Australian Special Interest Group for Computer Human Interaction. ACM, 167-176.

[4] Emiliano Castellina and Fulvio Corno. 2008. Multimodal gaze interaction in 3D virtual environments. COGAIN 8 (2008), 33-37.
[5] Matthieu Perreira Da Silva, Vincent Courboulay, and Armelle Prigent. 2007. Gameplay experience based on a gaze tracking system. In "Gaze-based Creativity, Interacting with Games and On-line Communities" INPROCEEDINGS in proceedings of COGAIN 2007 (Communication by Gaze Interaction IST FP6 European Project). $25-28$.

[6] Inger M Ekman, Antti W Poikola, and Meeri K Mäkäräinen. 2008. Invisible eni: using gaze and pupil size to control a game. In CHI'08 extended abstracts on Human factors in computing systems. ACM, 3135-3140.

[7] Massive Entertainment. 2019. Tom Clancy's The Division 2. Game.

[8] William W Gaver, Jacob Beaver, and Steve Benford. 2003. Ambiguity as a resource for design. In Proceedings of the SIGCHI conference on Human factors in computing systems. ACM, 233-240.

[9] Sébastien Hillaire, Anatole Lécuyer, Rémi Cozot, and Géry Casiez. 2008. Using an eye-tracking system to improve camera motions and depth-of-field blur effects in virtual environments. In Virtual Reality Conference, 2008. VR'08. IEEE. IEEE, $47-50$.

[10] Chien-Ming Huang, Sean Andrist, Allison Sauppé, and Bilge Mutlu. 2015. Using gaze patterns to predict task intent in collaboration. Frontiers in psychology 6 (2015), 1049.

[11] Poika Isokoski, Markus Joos, Oleg Spakov, and Benoît Martin. 2009. Gaze controlled games. Universal Access in the Information Society 8, 4 (2009), 323-337.

[12] Robert J. K. Jacob. 1990. What you look at is what you get: eye movement-based interaction techniques. In Proceedings of the SIGCHI conference on Human factors in computing systems: Empowering people (CHI '90). ACM, New York, NY, USA, 11-18. https://doi.org/10.1145/97243.97246

[13] Aleksandra Królak and Paweł Strumiłło. 2012. Eye-blink detection system for human-computer interaction. Universal Access in the Information Society 11, 4 (2012), 409-419.

[14] Michael Lankes, Bernhard Maurer, and Barbara Stiglbauer. 2016. An eye for an eye: Gaze input in competitive online games and its effects on social presence. In Proceedings of the 13th International Conference on Advances in Computer Entertainment Technology. ACM, 17.

[15] Michael Lankes, Thomas Mirlacher, Stefan Wagner, and Wolfgang Hochleitner. 2014. Whom are you looking for?: the effects of different player representation relations on the presence in gaze-based games. In Proceedings of the first ACM SIGCHI annual symposium on Computer-human interaction in play. ACM, 171179.

[16] Michael Lankes and Barbara Stiglbauer. 2016. GazeAR: Mobile gaze-based interaction in the context of augmented reality games. In International Conference on Augmented Reality, Virtual Reality and Computer Graphics. Springer, 397-406.

[17] Lennart Erik Nacke, Michael Kalyn, Calvin Lough, and Regan Lee Mandryk. 2011. Biofeedback game design: using direct and indirect physiological control to enhance game interaction. In Proceedings of the SIGCHI conference on human factors in computing systems. ACM, 103-112.

[18] Lennart E Nacke, Sophie Stellmach, Dennis Sasse, and Craig A Lindley. 2010. Gameplay experience in a gaze interaction game. arXiv preprint arXiv:1004.0259 (2010).

[19] Joshua Newn, Fraser Allison, Eduardo Velloso, and Frank Vetere. 2018. Looks can be deceiving: Using gaze visualisation to predict and mislead opponents in strategic gameplay. In Proceedings of the 2018 CHI Conference on Human Factors in Computing Systems. ACM, 261.

[20] Kher Hui Ng, Steve Benford, and Boriana Koleva. 2005. PINS push in and POUTS pop out: creating a tangible pin-board that ejects physical documents. In $\mathrm{CHI} 05$ Extended Abstracts on Human Factors in Computing Systems. ACM, 1981-1984.

[21] Kher Hui Ng, Boriana Koleva, and Steve Benford. 2007. The iterative development of a tangible pin-board to symmetrically link physical and digital documents. Personal and Ubiquitous Computing 11, 3 (2007), 145-155.

[22] J O'Donovan, J Ward, S Hodgins, and V Sundstedt. 2009. Rabbit run: Gaze and voice based game interaction. In Eurographics Ireland Workshop, December.

[23] Ken Pfeuffer, Jason Alexander, and Hans Gellersen. 2016. GazeArchers: Playing with Individual and Shared Attention in a Two-player Look\&Shoot Tabletop Game. In Proceedings of the 15th International Conference on Mobile and Ubiquitous Multimedia (MUM '16). ACM, New York, NY, USA, 213-216. https://doi.org/10. 1145/3012709.3012717

[24] Argenis Ramirez Gomez and Hans Gellersen. 2019. SuperVision: Playing with Gaze Aversion and Peripheral Vision. In Proceedings of the 2019 CHI Conference on Human Factors in Computing Systems. ACM, 473.

[25] Pedro Sanches, Kristina Hook, Corina Sas, and Anna Stahl. 2019. Ambiguity as a resource to inform proto-practices: The case of skin conductance. ACM Transactions on Computer-Human Interaction (TOCHI) (2019).

[26] C Schaefer, R Menges, K Schmidt, M Kuich, and T Walber. 2014. Schau genau! an eye tracking game with a purpose. Applications for Gaze in Games (2014).

[27] J David Smith and TC Graham. 2006. Use of eye movements for video game control. In Proceedings of the 2006 ACM SIGCHI international conference on Advances in computer entertainment technology. ACM, 20.

[28] India Starker and Richard A. Bolt. 1990. A Gaze-responsive Self-disclosing Display. In Proceedings of the SIGCHI Conference on Human Factors in Computing Systems (CHI '90). ACM, New York, NY, USA, 3-10. https://doi.org/10.1145/97243.97245

[29] Starbreeze Studios. 2013. Brothers: A Tale of Two Sons. Game. 
Exploring the Sensed and Unexpected:

Not Looking in Gaze Interaction

[30] Techland. 2018. Dying Light: Bad Blood. Game.

[31] Ubisoft Montreal and Ubisoft Toronto. 2018. Far Cry 5. Game [Xbox][Windows][PlayStation].

[32] MUHTAR ÇAĞKAN ULUDAĞLI and CENGİZ ACARTÜRK. 2018. User interaction in hands-free gaming: a comparative study of gaze-voice and touchscreen interface control. Turkish fournal of Electrical Engineering \& Computer Sciences 26, 4 (2018), 1967-1976.

[33] Boris Velichkovsky, Andreas Sprenger, and Pieter Unema. 1997. Towards gaze mediated interaction: Collecting solutions of the "Midas touch problem". In Human-Computer Interaction INTERACT'97. Springer, 509-516.

[34] Eduardo Velloso, Carl Oechsner, Katharina Sachmann, Markus Wirth, and Hans Gellersen. 2015. Arcade+: A Platform for Public Deployment and Evaluation of Multi-Modal Games. In Proceedings of the 2015 Annual Symposium on ComputerHuman Interaction in Play (CHI PLAY '15). ACM, New York, NY, USA, 271-275. https://doi.org/10.1145/2793107.2793145
HTTF 2019, November 19-20, 2019, Nottingham, United Kingdom

[35] Melodie Vidal. 2014. Shynosaurs: A Game of Attention Dilemma. In Proceeding of the First ACM SIGCHI Annual Symposium on Computer-human Interaction in Play (CHI PLAY '14). ACM, New York, NY, USA, 391-394. https://doi.org/10. $1145 / 2658537.2662979$

[36] Melodie Vidal, Remi Bismuth, Andreas Bulling, and Hans Gellersen. 2015. The Royal Corgi: Exploring Social Gaze Interaction for Immersive Gameplay. In Proceedings of the 33rd Annual ACM Conference on Human Factors in Computing Systems (CHI '15). ACM, New York, NY, USA, 115-124. https://doi.org/10.1145/ 2702123.2702163

[37] Tom Wilcox, Mike Evans, Chris Pearce, Nick Pollard, and Veronica Sundstedt. 2008. Gaze and voice based game interaction: the revenge of the killer penguins. SIGGRAPH Posters 81 (2008).

[38] Shumin Zhai, Carlos Morimoto, and Steven Ihde. 1999. Manual and gaze input cascaded (MAGIC) pointing. In Proceedings of the SIGCHI conference on Human Factors in Computing Systems. ACM, 246-253. 